\title{
Electroacupuncture decreases cognitive impairment and promotes neurogenesis in the APP/PS1 transgenic mice
}

\author{
Xuying $\mathrm{Li}^{1 \dagger}$, Fan Guo ${ }^{1 \dagger}$, Qiaomei Zhang ${ }^{1}$, Tingting Huo ${ }^{1}$, Lixin Liu ${ }^{2}$, Haidong Wei ${ }^{1}$, Lize Xiong ${ }^{1 *}$ and Qiang Wang ${ }^{1 *}$
}

\begin{abstract}
Background: Alzheimer's disease (AD) is a severe neurodegenerative disease for which there is currently no effective treatment. The purpose of this study was to investigate whether repeated electroacupuncture (EA) stimulation would improve cognitive function and the pathological features of $A D$ in amyloid precursor protein (APP)/presenilin 1 (PS1) double transgenic mice.
\end{abstract}

Methods: Cognitive function of APP/PS1 double transgenic mice was assessed using the Morris water maze test before and after EA treatment. Levels of amyloid $\beta$-peptide $(A \beta)$ deposits in the hippocampus and cortex were evaluated by immunofluorescence, western blot and enzyme-linked immunosorbent assay. Expression of brain-derived neurotrophic factor (BDNF) was also examined by immunofluorescence and western blot. The neurogenesis was labeled by the DNA marker bromodeoxyuridine.

Results: EA stimulation significantly ameliorated the learning and memory deficits of AD mice by shortening escape latency and increasing the time spent in the target zone during the probe test. Additionally, decreased A $\beta$ deposits and increased BDNF expression and neurogenesis in the hippocampus and cortex of EA-treated AD mice were detected. The same change was detected in wild-type mice after EA treatment compared with wild-type mice without EA treatment.

Conclusions: Repeated EA stimulation may improve cognitive function, attenuate $A \beta$ deposits, up-regulate the expression of BDNF and promote neurogenesis in the APP/PS1 double transgenic mice. This suggests that EA may be a promising treatment for AD.

Keywords: Alzheimer's disease, Electroacupuncture, Neurogenesis, BDNF, Aß deposits

\section{Background}

Alzheimer's disease (AD) is one of the most common neurodegenerative diseases. Its main clinical manifestations include dementia, memory loss, personality disorders and language problems. The global prevalence of dementia was estimated to be as high as 24.2 million by 2005 , and about $70 \%$ of these cases were attributed to $\mathrm{AD}$ [1]. Prevalence is predicted to reach 80 million worldwide by 2040 [2]. Confronted with such a large number of people suffering from AD, the currently available treatments for the disease are limited and without

\footnotetext{
* Correspondence: |xiong@fmmu.edu.cn; wangqiang@fmmu.edu.cn

${ }^{\dagger}$ Equal contributors

${ }^{1}$ Department of Anesthesiology, Xijing Hospital, The Fourth Military Medical University, Xi'an 710032, Shaanxi Province, China
}

Full list of author information is available at the end of the article curative effects. Therefore, identifying effective and safe treatment with clear fundamental mechanisms is urgently needed.

The pathology of AD is generally accepted as being characterized by the abnormally abundant deposition of amyloid plaques, neurofibrillary tangles, and selective neuronal loss in the frontal and temporal cortices, as well as the hippocampus of brain. The accumulation of amyloid $\beta$-peptide $(\mathrm{A} \beta)$ plays the most important role in the pathogenesis of AD. A wealth of evidence has indicated that $A \beta 1-42$ deposits participate in the process of neuron loss and lead to the occurrence of dementia in $\mathrm{AD}$ patients.

$\mathrm{AD}$ is a multifactorial disease, and a decrease in neuro-regenerative capacity is an important factor in the 
decline of neural plasticity, development of $\mathrm{A} \beta$ plaques and neurofibrillary tangles [3]. Numerous studies have provided powerful evidence that the decline of hippocampal neurogenesis participates in the development of $\mathrm{AD}$ and induces impairment of learning and memory [4]. Several studies have indicated that brain-derived neurotrophic factor (BDNF) is an important factor for promoting neurogenesis in the adult central nervous system under physiological or pathological conditions $[5,6]$. Moreover, some clinical studies have revealed that the level of BDNF expression is significantly decreased in the hippocampus and some cortical areas of AD patients $[7,8]$. These data suggest that up-regulation of BDNF and promotion of neurogenesis would be a promising target for $\mathrm{AD}$ treatment.

Electroacupuncture (EA), a traditional Chinese medicine treatment that stimulates certain acupoints, has been shown to induce significant neuroprotective effects in various kinds of central nervous system diseases, as well as improve neuroethology [9-11]. The mechanism for EA improving neurological deficits in ischemic injury is proven to be through the promotion of neurogenesis [12]. EA has also been proven to enhance BDNF activation in the dentate gyrus in rats [13]. However, whether EA stimulation is effective for AD still remains unclear.

In the present study, we investigated whether EA treatment could ameliorate cognitive impairment and attenuate $\mathrm{A} \beta$ deposits, and the effect of EA treatment on BDNF expression and neurogenesis in the amyloid precursor protein (APP)/presenilin 1 (PS1) double transgenic $(\mathrm{Tg})$ mice.

\section{Methods}

Animals

We used an APP/PS1 double Tg C57BL/6 J mouse model, which could effectively simulate the pathological features of $\mathrm{AD}$ patients. We used this model to investigate the effect of EA treatment on AD mice. Male APP/ PS1 double Tg mice (2-months old) were purchased from Beijing HFK Bioscience Co. LTD (Beijing, China) and randomly divided into two groups: an APP/PS1 group (APP) and an EA treatment APP/PS1 group (APP + EA). Normal male C57BL/6 J mice (2 months old) were obtained from the Experimental Animals Center of the Fourth Military Medical University (Xi'an, China) and randomly divided into two groups: a control group (Con) and an EA treatment control group (Con + EA). Animals were housed under controlled temperature $\left(25^{\circ} \mathrm{C}\right)$, 12-hour light/dark cycles and allowed free access to water and food. All experiments were carried out according to the Guidelines for Animal Experimentation of the Fourth Military Medical University. The experimental protocol was approved by the Ethics Committee for Animal Experimentation, and was performed according to the
Guidelines for Animal Experimentation of the Fourth Military Medical University and to the National Institute of Health Guide for the Care and Use of Laboratory Animals.

\section{EA treatment}

In line with our previous studies, we performed EA treatment at the "Baihui (GV20)" acupoint with an intensity of $1 \mathrm{~mA}$ and frequency of $2 / 15 \mathrm{~Hz}$ for four weeks for total 20 days (30 min/day, 5 days/week) [10,14]. We used an EA instrument (Hwato, model No. SDZ-V, Suzhou Medical Instruments Co, Ltd, Suzhou, China) and selected the dense-sparse wave type. During EA treatment, we maintained the rectal temperature of all animals at $37.0 \pm 0.5^{\circ} \mathrm{C}$ and we provided inhaled oxygen by facemask at a flow rate of $1 \mathrm{~L} / \mathrm{min}$. The animals in control group received no treatment.

\section{Morris water maze test}

Following the methods previously described, we used the Morris water maze (MWM) test to evaluate learning and memory impairment in each group in this experiment. We evaluated the Morris water maze test at 7 months and 10 months (before EA treatment), and at 11 months (immediately after EA treatment). The apparatus consisted of a circular pool $(120 \mathrm{~cm}$ diameter $\times 50 \mathrm{~cm}$ height) with a black inner wall, which was subdivided into four equal quadrants and filled with water $\left(25^{\circ} \mathrm{C}\right)$ to the depth of $30 \mathrm{~cm}$. An escape platform $(10 \mathrm{~cm}$ diameter) was placed in one of the quadrants (the target quadrant) and submerged approximately $2 \mathrm{~cm}$ below the surface of the water. Mice were released into the water facing the wall of the pool. The test contained a platform trial that measured the animal's spatial acquisition ability and a spatial probe test that assessed memory. On the first day, the mice in each group performed four platform trials with the platform submerged in water in the same place each time. They then performed four training trials per day for five days. Finally, 24 hours after the $5^{\text {th }}$ day, a probe test, where the platform was removed, was performed. All the data, including the swim path and the swim time, were measured by an automated analyzing system (Dig-Behav, Jiliang Co., Ltd., Shanghai, China).

\section{Sample preparation and double-label immunofluorescence assays}

Immunofluorescence was used to evaluate levels of $A \beta$ deposits and BDNF expression in the hippocampus and cortex of each group. At first, to remove the blood and fix the brain tissues, the Tg mice were deeply anesthetized with $0.8 \%$ pentobarbital sodium and perfused with physiological saline, followed by $4 \%$ (v/v) ice-cold paraformaldehyde in PBS $(\mathrm{pH}=7.4)$. Brain tissues were then harvested and postfixed for 2 hours in the same fixative 
at $4^{\circ} \mathrm{C}$ and cryoprotected in $20 \%$ and $30 \%$ sucrose solutions. Coronal Sections $12 \mu \mathrm{m}$ in thickness were cut using a cryostat and block with PBS containing 0.3\% (v/ v) Triton X-100 and 3\% (v/v) normal goat serum. To verify the success of the $A D$ model building, the tissue sections were incubated overnight with rabbit anti-A $\beta$ primary antibodies (1:400, Cell Signaling Technology, Inc. Danvers, MA, USA) followed by the anti-rabbit Cy3 tagged secondary antibodies (1:200; CWBIO, Beijing, China). To investigate the effect of $E A$ on $A \beta$ deposits and BDNF expression in the brain, immunofluorescence was conducted. The sections were incubated with the first antibody of rabbit anti-BDNF (1:200, Beijing Biosynthesis Biotechnology Co., Ltd, Beijing, China) or rabbit anti-A $\beta$ antibody (1:400, Cell Signaling Technology, Inc. Danvers, MA, USA) followed by the anti-rabbit Cy3 tagged secondary antibodies (1:200; CWBIO, Beijing, China). The sections were mounted with $50 \%$ glycerol for examination under a fluorescence microscope. Images were observed and captured with a co-focal laser microscope (FV1000; Olympus BX51; Tokyo, Japan).

\section{Western blot analysis}

Animals of each group were anesthetized with sodium pentobarbital (100 mg/kg, i.p.) and sacrificed immediately by decapitation. Brain tissues were then removed, weighed and homogenized in RIPA buffer (Beyotime P0013C, Haimen, Jiangsu, China) plus protease inhibitors. A BCA Protein assay kit (Beyotime P0012S, Haimen, Jiangsu, China) was used to determine the protein concentrations of each sample; 40ug protein samples were separated by 15\% SDS-PAGE. The proteins were then transferred onto polyvinylidene difluoride membrane. After blocking with $3 \%$ nonfat milk, membranes were incubated with the following primary antibodies: rabbit anti-BDNF monoclonal antibody (1:100, Beijing Biosynthesis Biotechnology Co., Ltd, Beijing, China), rabbit anti- $\beta$-actin (1:10000, Epitomics, Inc., P60709), and rabbit anti-A $\beta$ (1:400, Cell Signaling Technology, Inc. Danvers, MA, USA). Subsequently, membranes were incubated for 1 hour at room temperature with secondary antibody of antirabbit HRP-conjugated IgG (1:20000, CWBIO, Beijing, China). Labeled protein was detected using chemiluminescence reagents (ECL; Amersham Bio-sciences, Little Chalfont, Buckinghamshire, UK) and the band intensity was analyzed (Alpha Innotech).

\section{Quantification of $A \beta 1-42$ level by ELISA}

Levels of soluble $A \beta 1-42$ in the hippocampus and cortex of $\mathrm{Tg}$ mice were determined with sandwich enzyme-linked immunosorbent assay (ELISA), using mouse $\beta$-Amyloid (1-42) ELISA kit (Westang Ltd, Shanghai, China). According to the manufacturer's instruction, brain tissues were homogenized in RIPA buffer (Beyotime P0013C, Haimen, Jiangsu, China) and centrifuged at $27,000 \times \mathrm{g}$ at $4^{\circ} \mathrm{C}$ for $30 \mathrm{~min}$ to obtain the supernatants. Then, the standards and samples which were mixed with specific first antibody in duplicate were added to the microtiter wells. HRPconjugated secondary antibody was added to the plates for $1 \mathrm{~h}$ at room temperature after extensive washing, followed by chromogen for 15-30 min. The enzymatic reaction was then terminated by addition of a stop solution (2 $\mathrm{N} \mathrm{H}_{2} \mathrm{SO}_{4}$ ). Optical density (OD) was read at $450 \mathrm{~nm}$ within $30 \mathrm{~min}$ on a microplate spectrophotometer (Denley Dragon Wellscan MK 3). Concentrations were calculated according to the standard curve.

\section{BrdU staining}

The newly born neurons were labeled by DNA marker Bromodeoxyuridine (BrdU) (Sigma, St. Louis, MO, dissolved in $0.9 \% \mathrm{NaCl}$ ). BrdU was administered intraperitoneally at a concentration of $100 \mathrm{mg} / \mathrm{kg} /$ day by injection for seven consecutive days. And the first administration was at the last 2 rest day of $4^{\text {th }}$ cycle of the electroacupuncture. To label differentiated neurons in the hippocampus and cortex, 11-month-old Tg mice $\mathrm{APP}, \mathrm{APP}+\mathrm{EA}$ and nontransgenic mice Con, Con + EA $(\mathrm{n}=5$ each) were sacrificed 28 days after the last injection of BrdU. The mice were then anesthetized and perfused with phosphate-buffered saline following by $4^{\circ} \mathrm{C} 4 \%$ paraformaldehyde. The brain was removed and postfixed in buffered 4\% paraformaldehyde for 2 hours. After being immersed in $20 \%$ and $30 \%$ sucrose solution, the brains were cut into $12 \mu \mathrm{m}$-thick coronal sections.

To label the relative distribution of the new proliferating cells, BrdU + Nissl double staining was used. First, sections of every group were incubated with $10 \%$ normal goat serum in $0.1 \mathrm{M} \mathrm{PBS} 60 \mathrm{~min}$ at $37^{\circ} \mathrm{C}$. The primary antibodies Nissl (Abcam) and rat anti-BrdU (ab6326, Abcam LTD., Hongkong, China) were used. After washing in PBS, the sections were incubated in secondary antibody Biotinylated Goat Anti-body IgG antibody (1:300, Vector, BA 9400), and the third antibody Cy3conjugeted streptavidin (1:800). For each group, brain sections starting from the bregma -2.1 and ending at the bregma -4.5 were selected for counting of the $\operatorname{BrdU}(+)$ and $\operatorname{Nissl}(+)$ cells. The $\operatorname{BrdU}(+)$ and $\mathrm{Nissl}(+)$ cells were calculated using a $\times 400$ magnification in the DG, CA1 regions.

\section{Statistical analysis}

SPSS 14.0 for Windows was used to conduct the statistical analyses. Two-way analysis of variance (ANOVA) with repeated measures was used for analyzing data from the Morris water maze test. Other statistical tests were performed using one-way ANOVA and Student's 
t-test for comparisons. The $P$ values of $\leq .05$ were considered indicative of statistical significance. All date are expressed as mean \pm standard deviation (SD).

\section{Results}

Cognitive impairment and $A \beta_{1-42}$ deposition presented in the APP/PS1 mice

Transgenic mice, which imitated the most salient characteristics of $\mathrm{AD}$, were selected to simulate human Alzheimer's disease. The effectiveness of the Tg mouse model to mimic AD was evaluated using a neurological behavior test (Morris water maze test) and a pathology test (immunofluorescence that specifically identified a biomarker of AD) of 7-month-old and 10-month-old Tg mice. Spatial learning and memory function performance were more severely damaged in the 7-month-old group of double $\mathrm{Tg}$ mice than in the wild-type C57 mice; this was evaluated by the platform trial and spatial probe test of the Morris water maze test (Figure 1A, B) without a significant difference in swimming speed (Figure $1 \mathrm{C}$ ). However, there was no significant difference between the 7-month-old and 10-month-old Tg mice groups. This indicated that the pathological features of APP/PS1 double Tg mice developed from at least 7 months and remained stable.

Total A $\beta$ deposits in the hippocampus and cortex were detected by quantitative immunofluorescence in different groups (Figure 1D). The $A \beta$ level of the hippocampus and cortex in the 10-month-old group was increased compared with wild-type group.

\section{EA ameliorated cognitive impairment in APP/PS1 double Tg mice}

We investigated the effects of EA stimulation on cognitive function through use of the Morris water maze (MWM) test. This test was conducted following the procedure diagram shown in Figure 2A. As shown in Figure $2 \mathrm{~B}-\mathrm{C}$, escape latency indicated that the APP + EA group had better cognitive performance than the APP group. Cognitive impairment in the different groups was confirmed in the probe trail, which showed that the $\mathrm{APP}+\mathrm{EA}$ group and the Con + EA group mice spent more time in the target quadrant than their non EA-treated counterparts $(P<.05)$. This difference was not attributable to the presence of motor deficits as the four groups of mice exhibited similar swimming speeds (Figure 2D). Taken together, the above results demonstrate that EA stimulation significantly improved learning and memory functioning in both APP/PS1 Tg mice and wild-type mice.

\section{EA reduced brain $A \beta_{1-42}$ deposition in APP/PS1 double Tg mice}

To investigate the effect of EA on $A \beta_{1-42}$ deposition in the hippocampus and cortex, three methods, including immunofluorescence staining, ELISA and western blots, were used. As shown in Figure $3 A-B, A \beta_{1-42}$ staining (red) was co-localized with neurons (Nissl staining, green) in all studied regions. The APP + EA group exhibited notable reductions in $A \beta_{1-42}$ deposits compared with the APP group, in both the hippocampus and
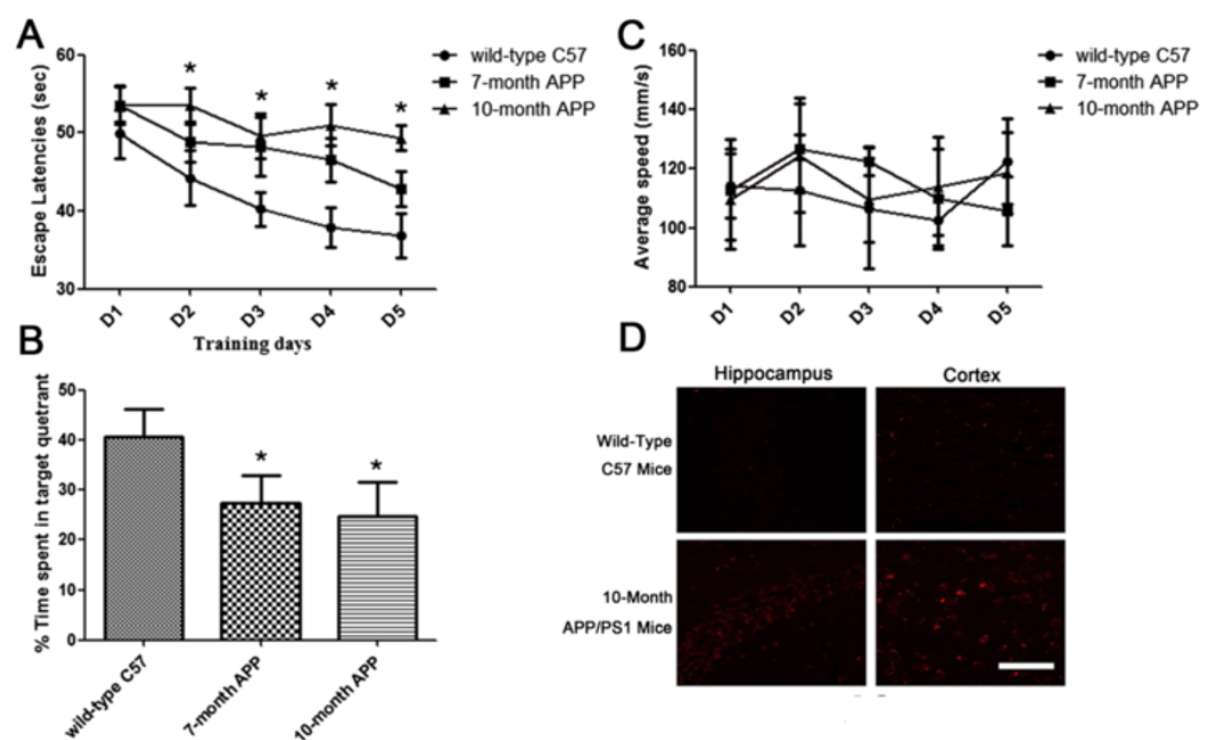

Figure 1 APP/PS1 mice demonstrated features of cognitive impairment seven months after birth. (A) 7-month-old and 10-month-old APP/PS1 mice showed longer latencies for reaching the platform in the Morris Water Maze test than the wild-type mice. (B) The average swimming speeds among different groups were not significant different. (C) During the probe trail, wild-type mice spent more time in the target quadrant than 7-month-old and 10-month-old APP mice. ( $n=6$ in each group) ${ }^{*} P<.05$ from wild-type mice); (D) Compared with the wild-type mice, total A $\beta$ deposits in the hippocampus and cortex of 7-month-old and 10-month-old APP mice were increased. 

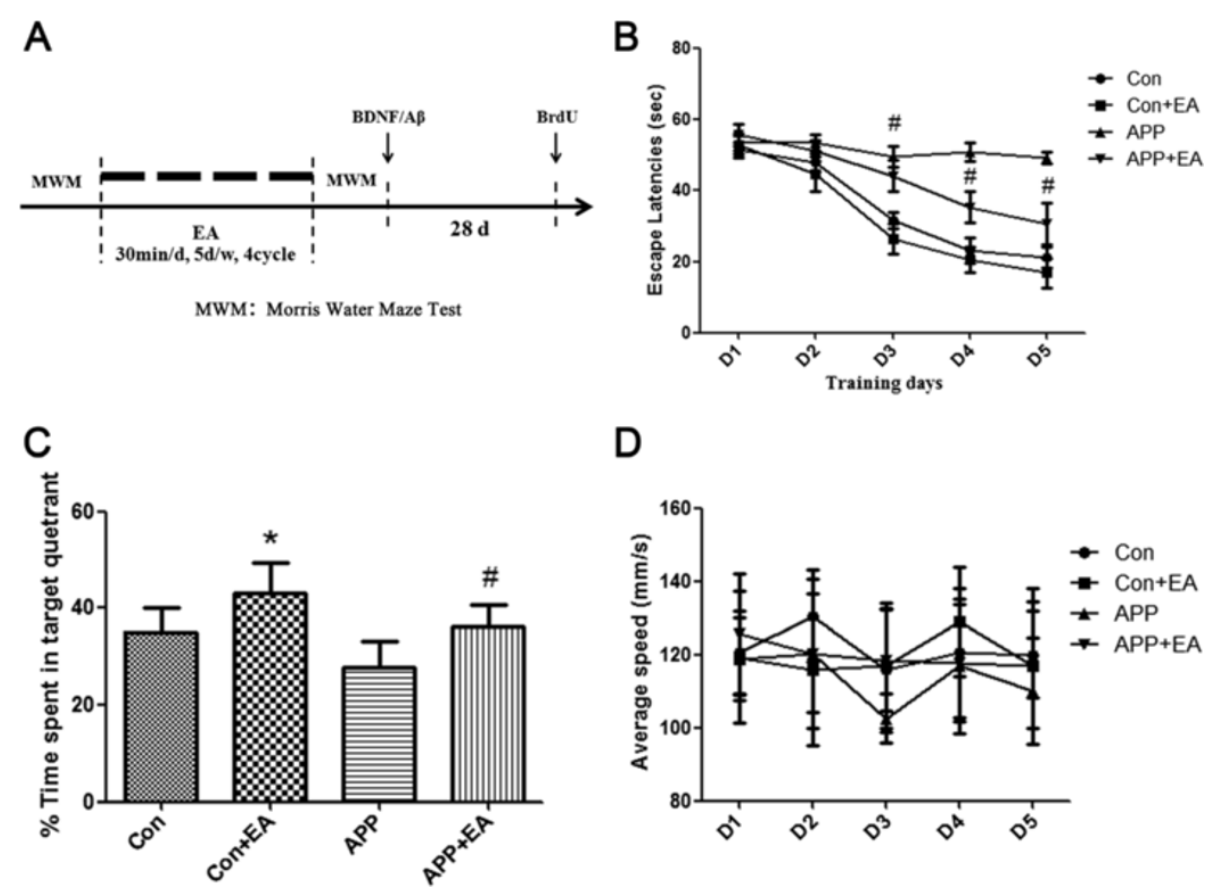

Figure 2 Electroacupuncture ameliorated cognitive impairments in APP/PS1 mice. (A) Schematic representation of the experiment protocol. (B) Mice from the APP group showed longer latencies for reaching the platform than those from the APP + EA group. (C) The probe test indicated that the time spent in the target quadrant was increased in the APP + EA group and Con + EA group compared with their non EA-treated counterparts. (D) No significant difference was detected in average swimming speed among all the groups. ( $n=6$ in each group) ( ${ }^{*}<.05$ vs. Con group; $\# P<.05$ vs. APP group).

cortex. Meanwhile, EA stimulation also reduced the deposit of $A \beta_{1-42}$ in normal-aged mice compared with the Con group.

ELISA test results showed that the levels of $A \beta_{1-42}$ deposits from the hippocampus and cortex in the APP + EA group and the Con + EA group were significantly lower than those in their non EA-treated counterparts $(P<.05$; Figure 4A-B). At the same time, gray-scale analysis of western blot bands indicated that the expression of $A \beta_{1-42}$ from the APP + EA group was lower than that in the APP group (Figure $4 \mathrm{C}-\mathrm{D}$ ). A similar result was detected in the Con group when compared with the Con + EA group. These results demonstrate that long-term EA treatment is an effective method for inhibiting the deposition of $\mathrm{A} \beta_{1-42}$.

\section{EA increased BDNF expression in APP/PS1 double Tg mice}

The results of immunofluorescence with the antibody specifically recognizing BDNF in the brain showed significantly stronger expression in both the hippocampus and cortex from the EA-treated groups than that in the non EA-treated groups (Figure 5A-B). Western blot results also confirmed that the expression of BDNF was up-regulated in the APP + EA and Con + EA groups compared with their non EA-treated counterparts (Figure $5 \mathrm{C}, \mathrm{P}<.05$ ).

\section{EA promoted neurogenesis in APP/PS1 double Tg mice}

Survival and differentiation of the newborn cells to neurons $(\mathrm{BrdU}+\mathrm{Nissl}+)$ were detected 28 days after the last injection of BrdU. As shown in Figure 6, the result of $\mathrm{BrdU}(+)$ and $\mathrm{Nissl}(+)$ cells was consistent with the change of BDNF expression in the brain. There was a significant decrease in $\mathrm{BrdU}(+)$ and $\mathrm{Nissl}(+)$ cells in the APP/PS1 Tg mice groups (including the APP and APP + EA groups) when compared with the wild-type group (including Con and Con + EA groups $)(P<.05)$. EA treatment also significantly promoted neurogenesis in the APP + EA group, as well as in the Con + EA group, compared with their non EA-treated counterparts.

\section{Discussion}

$\mathrm{AD}$ is a neurodegenerative disease that seriously affects the quality of life for thousands of patients. There is also an enormous economic impact of $\mathrm{AD}$ for governments. In Europe, the total costs for dementia in 2008 were estimated to be more than 177 billion Euros [15], which exceeded the costs for patients with either cancer or cardiovascular diseases [2]. The existing treatments for $\mathrm{AD}$ are unsatisfactory, and searching for an effective 

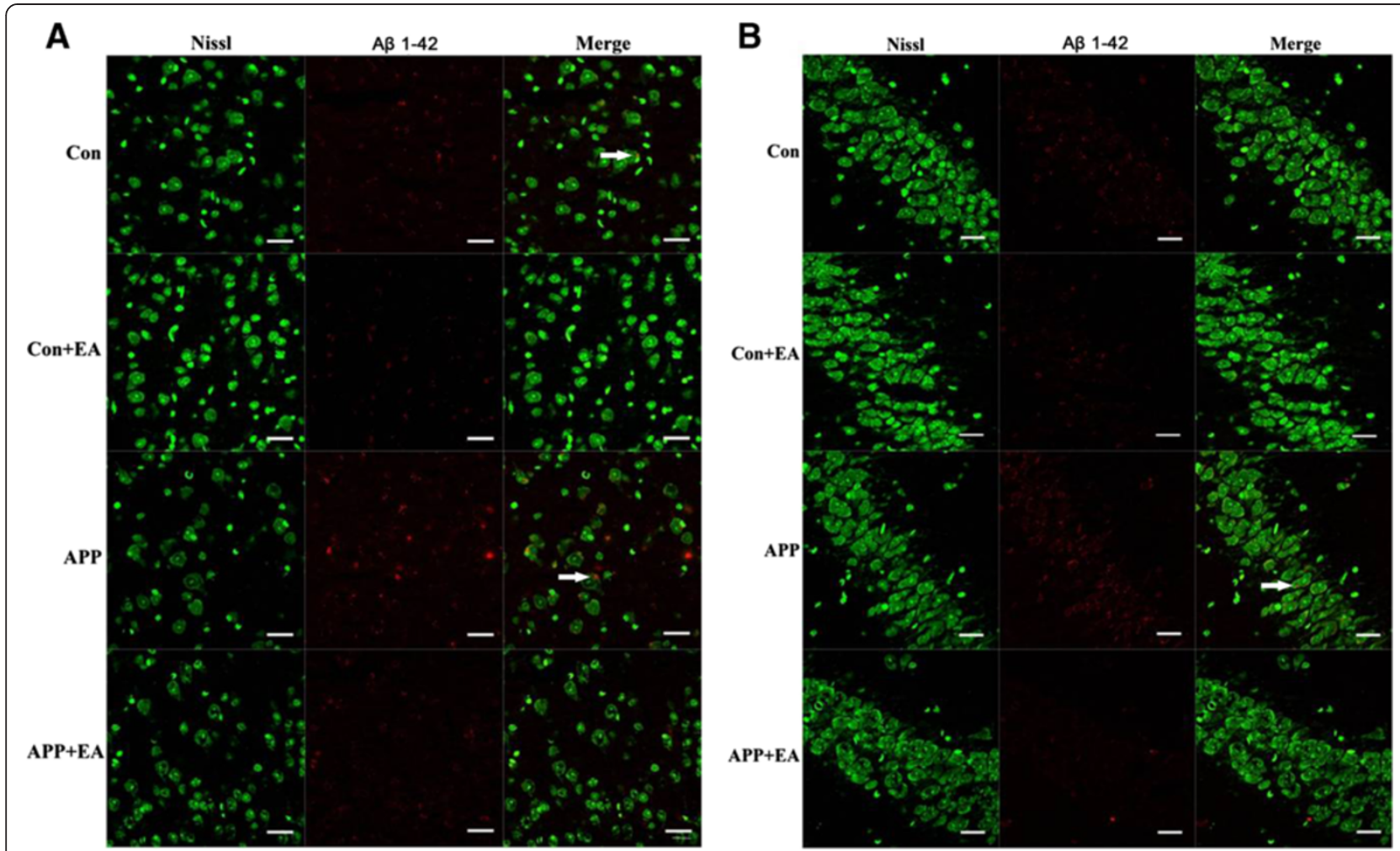

Figure 3 Electroacupuncture suppressed the deposition of $A \beta_{1-42}$ in the hippocampus and cortex. With immunofluorescence staining following the EA administration, compared with the APP group, the deposition of AB1-42 was significantly decreased in the APP + EA group in the (A) cortex and (B) hippocampus. Bar $=20 \mu \mathrm{m}$.

treatment method for patients suffering from $\mathrm{AD}$ is a major challenge. In the present study, we confirmed the hypothesis that repeated EA stimulation improved cognitive functioning and reduced the accumulation of $A \beta_{1-42}$ deposits in the brain. Moreover, the beneficial effects accompanied with the up-regulation of BDNF expression and promotion of neurogenesis in the APP/PS1 double $\mathrm{Tg}$ mice, suggest that EA may be a promising treatment for AD.

In the last two decades, several kinds of genetically modified mice have been generated as potential models for studying neurodegenerative processes [16], such as PDAPP mice reported by Masliah et al. [17] and Tg2575 mice reported by Shi et al. [18]. In the current study, we chose the model of APP/PS1 double $\mathrm{Tg}$ mice, which were generated by knocking identified familial $\mathrm{AD}$ and/ or PS1 into the mice genome; this model has been widely adopted by other scientists. The APP/PS1 double $\mathrm{Tg}$ mouse was used as the AD model for its aggressive, early-onset brain amyloidosis, as well as the concurrent atrophy and substantial cell loss. The clinical relevance of this model is supported by disturbances of neuronal structure in the form of dystrophic neurites surrounding plaques, decreased fiber density, and synaptic dysfunction that imitates most aspects of AD brain pathology
$[19,20]$. Thus, the APP/PS1 double Tg mouse model is the closest representative of AD pathology. In this study, the effectiveness of the $\mathrm{Tg}$ mouse model to mimic $\mathrm{AD}$ was evaluated through neurological behavior and pathology assessment. Cognitive impairment presented in 7 -month-old double $\mathrm{Tg}$ mice, and the $\mathrm{A} \beta$ deposits in the hippocampus and cortex were detected in 10-month-old double $\mathrm{Tg}$ mice. These findings indicate that the pathological features of APP/PS1 double Tg mice that mimic $\mathrm{AD}$ remained stable.

Classic and well-known symptoms of AD include problems with spatial learning and presence of a memory deficit. Previous studies have shown that EA stimulation can protect against neuronal damage, and effectively prevent the impairment of learning and memory caused by cerebral ischemia injury or high-sustained positive acceleration $(+\mathrm{Gz})$ exposures $[9,21]$. Our study demonstrated that EA stimulation significantly restored spatial learning and memory function of AD mice. This suggests that that EA stimulation may be effective in potentially ameliorating cognitive impairment caused by AD.

The identification of reliable biomarkers has been hindered by the fact that the diagnosis of $\mathrm{AD}$ in clinical practice depends largely on a patient's symptoms. However, increasingly accurate pathological diagnostic methods have 


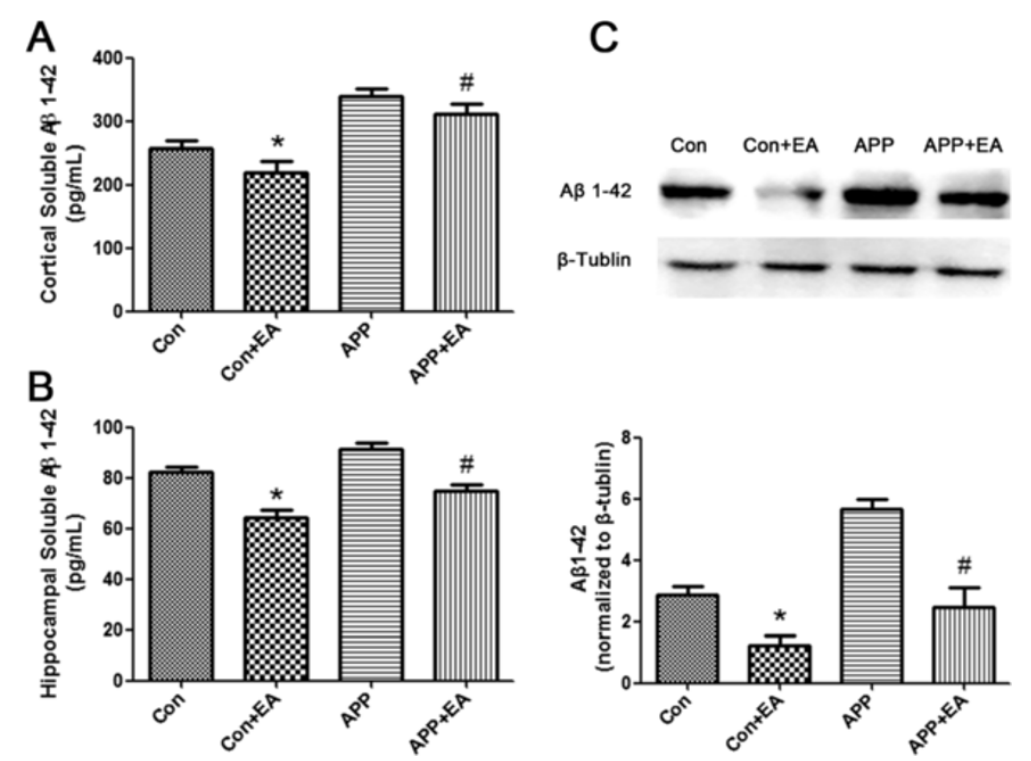

Figure 4 Electroacupuncture suppressed the deposition of $A \beta_{1-42}$ in the hippocampus and cortex. ELISA test results revealed that $A \beta 1-42$ depositions from the (A) cortex and (B) hippocampus in the APP + EA group and the Con + EA group were significantly lower than those in their non EA-treated counterparts ( $n=5$ in each group) $\left({ }^{*} P<.05\right.$ vs. Con group; $\# P<.05$ vs. APP group). (C) Western blot result indicated that the expression of $A \beta_{1-42}$ in the Con + EA group was lower than that in Con group. A similar result was detected in the APP + EA group compared with the APP group. ( $n=3$ in each group) ( ${ }^{*} P<.05$ vs. Con group; $\# P<.05$ vs. APP group).

become a reality because of the identification of biomarkers such as APP, A $\beta$, tau and p-tau, isoprostanes, and inflammatory makers. Among these, $\mathrm{A} \beta$ deposits are the most typical pathological sign and a defining factor for cognitive impairment in AD brains. In the brain, two different kinds of $A \beta$ exist ( $A \beta 1-40$ and $A \beta 1-42)$. $A \beta 1-42$, the metabolite of the APP and PS1 gene mutation, is a major component of senile plaques [22-24]. Additionally, the accumulation of $A \beta 1-42$ in the brain is more cytotoxic than $A \beta 1-40$, in the context of $\mathrm{AD}$. $\mathrm{A} \beta$ is not only a biomarker for diagnosing
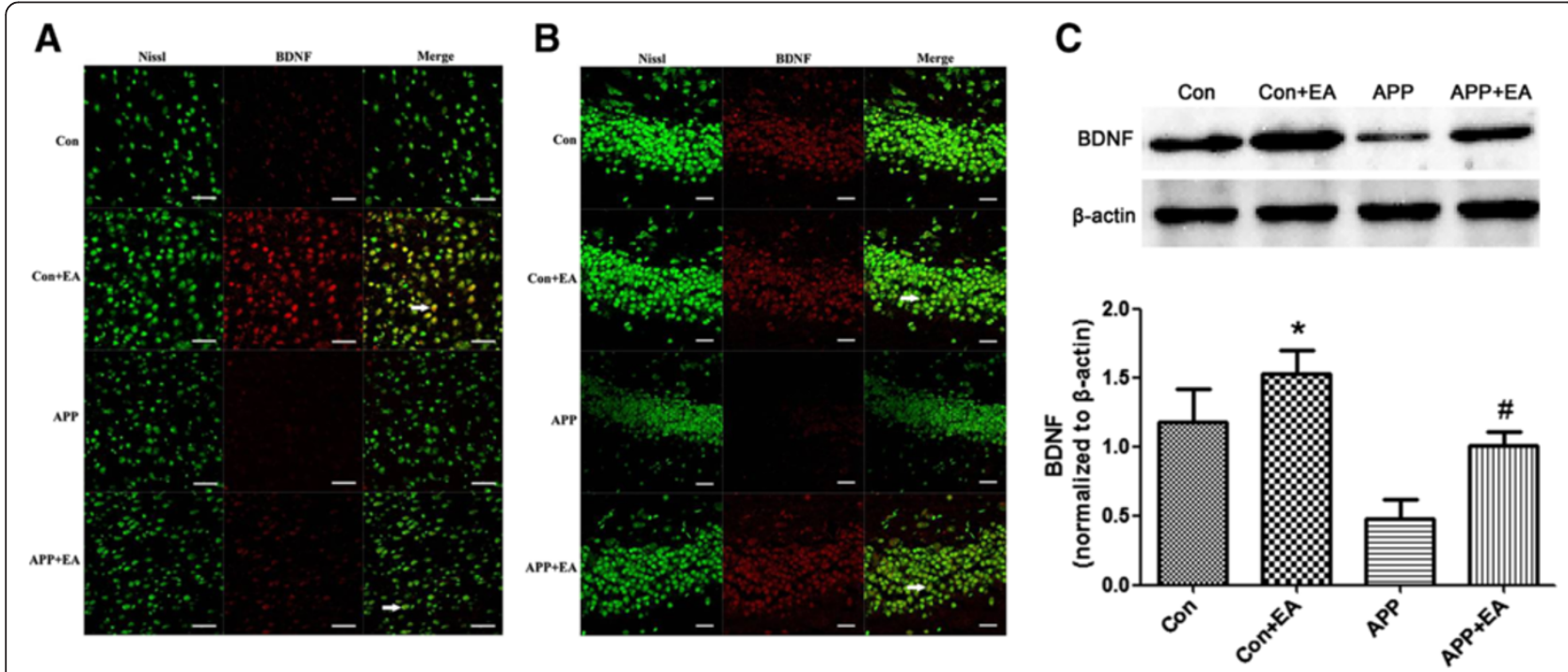

Figure 5 Electroacupuncture upregulated the expression of BDNF in hippocampus and cortex. Representative double

immunofluorescence staining of BDNF (red) and NeuN (green) in the (A) cortex and (B) hippocampus. With EA treatment, BDNF was upregulated in the APP + EA group and Con + EA group compared with their non EA-treated counterparts ( ${ }^{P}<.05$ vs. Con group; $\# P<.05$ vs. APP group). (C) The protein expression of BDNF was evaluated using western blot. EA significantly up-regulated the expression of BDNF in the APP/PS1 mice and wild-type mice compared with their matched counterparts. Data are means $\pm S D\left(n=5\right.$ in each group) $\left({ }^{*} P<.05\right.$ vs. Con group; $\# P<0.05$ vs. APP group). $\operatorname{Bar}=20 \mu \mathrm{m}$. 


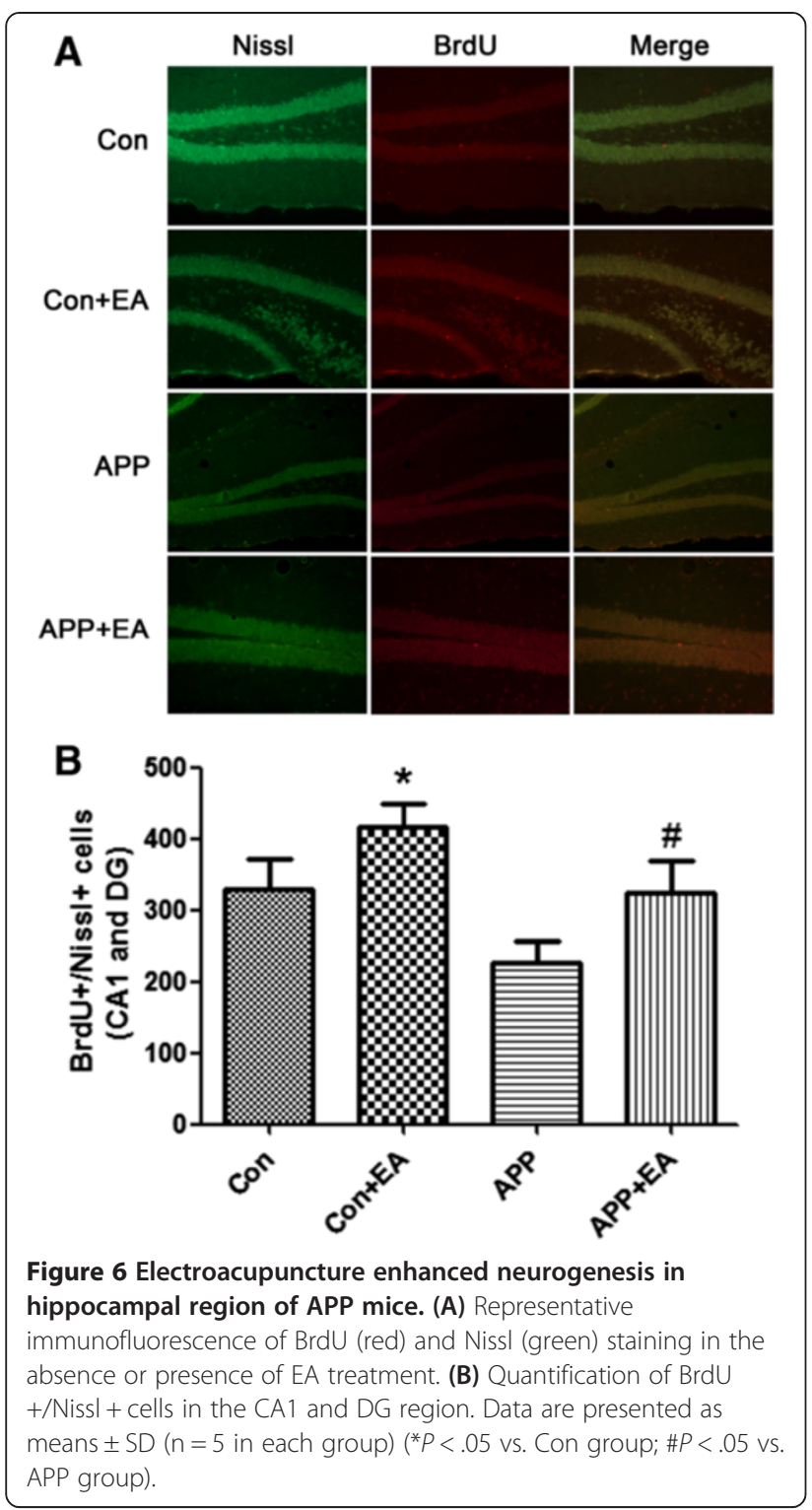

AD; it is also an important target for AD therapy. Application of an antagonist of $A \beta$ has been shown to improve memory impairment in APP $\mathrm{Tg}$ mice [25]. Thus, researchers are focusing on finding effective methods to lower $A \beta$ deposits, especially those of $A \beta 1-42$, to reverse the pathological features of AD. In our study, we demonstrated that EA treatment was a feasible and effective way for lowering A $\mathrm{B} 1-42$ deposits in APP/PS1 mice. However, the mechanism for EA to reduce $A \beta$ deposit is still unclear and needing further exploration.

The subventricular zone of the lateral ventricle and the subgranular layer of the dentate gyrus in the hippocampus are two parts associated with adult neurogenesis. Dramatic decline in neurogenesis has been proven to contribute to the impairments of learning and memory in AD models [26,27]. Some key proteins involved in
AD pathogenesis, including apolipoprotein E4 (APOE4), APP and PS1, have been proved to regulate neurogenesis [28-30]. Regenerative medicine, which is different from slowing down or stopping the progression of $\mathrm{AD}$, may offer a new therapeutic strategy for patients. The newborn neurons may enhance neuronal plasticity and integrate into existing neural circuits physically and functionally [4]. Our results revealed that with BrdU staining, repeated EA treatment could enhance neurogenesis in APP/PS1 Tg mice. The decrease in A $\beta 1-42$ that we mentioned above may be an important factor for nerve regeneration.

The mammalian neurotrophin family, including nerve growth factor, BDNF and neurotrophin-3, activates different cell signaling pathways via tyrosine receptor kinase. BDNF localizes in the hippocampus, hypothalamus, cortex, septum and the adrenergic brain stem nuclei of the brain and participates in neuron development, differentiation and plasticity maintenance throughout life [31]. BDNF plays an important role in memory formation and storage by regulating synaptic plasticity. Even if there have been conflicting results regarding the expression of BDNF in AD patients [32], most of the results indicate that BDNF is severely decreased in the hippocampus and some cortical areas [7,33]. Meanwhile, BDNF could reduce cellular damage caused by A $\beta 1-42$ [34]. Our results showed that EA treatment significantly increased the expression of BDNF in both the hippocampus and cortex; this suggests that the increase in BDNF may be involved in the therapeutic effect of EA for AD. From our current study, the expression of $A \beta$ and BDNF changing were also observed in wild-type mice in. We assumed that the effect of EA on $A \beta$ and BDNF could be related to a common signaling pathway but not a specific pathway in AD. Moreover, the control group was age-matched with the APP mice. Our result might suggest that electroacupuncture could induce the protective effect for the central nervous system of aging and Alzheimer's disease.

In the present study, we observed that EA stimulation significantly improved the neurological behavior performance of $\mathrm{AD}$ mice, and reduced the deposition of $\mathrm{A} \beta$ in the hippocampus and cortex. At the same time, a noticeable increase in neurogenesis and BDNF expression in the hippocampus and cortex was also detected. Thus, our preliminary presumption was that EA stimulation improved neurobehavioral performance through promoting neurogenesis and BDNF expression in the brain. However, the link between neurogenesis and the pathology of $\mathrm{AD}$ is still not fully understood. It is proven that $A \beta$ deposits impair the transduction path activated by BDNF such as Ras/ERK, PI3K/Akt and PKA/CREB [35,36]. Moreover, BDNF is required for long-term survival of newborn neurons [37]. Presumably, EA may 
affect BDNF and its downstream pathway to induce neurogenesis, and improve neurobehavior in an $\mathrm{AD}$ model. The precise mechanism of EA treatment for $\mathrm{AD}$ still needs further elucidate.

In addition, Baihui (GV20) acupoint belongs to the Du series. According to traditional Chinese medical theory, acupoint of the Du series is the first choice in treating brain disease. Our previous experimental studies [9-11,38] have proved that EA at the Baihui acupoint protects brain from ischemic injury. Thus, we chose the Baihui acupoint in the present study. Moreover, our previous study [14] also investigated the specificity of Baihui acupoint in a rat cerebral ischemic model. Although in the current paper we aimed to focus proving that EA owned the possibility for improving cognitive dysfunction, the specificity for EA treatment in AD model still need further exploration. This is a limitation of our study, a sham-acupuncture considered as a control is necessary for future study.

\section{Conclusions}

In summary, the present study demonstrated that EA stimulation in Baihui (GV 20) acupoint ameliorated learning and memory deficits and reduced $\mathrm{A} \beta 42$ deposit in APP/PS1 mice. We propose that the underlying mechanism of EA may be related to the promotion of neurogenesis and the up-regulation of BDNF expression in the hippocampus and cortex. These findings provide strong evidence for EA as a novel strategy for AD treatment.

\section{Abbreviations \\ EA: Electroacupuncture; APP/PS1: Amyloid precursor protein/presenilin 1; AD: Alzheimer's disease; A $\beta$ : Amyloid $\beta$-peptide; BDNF: Brain-derived neurotrophic factor; BrdU: Bromodeoxyuridine; Tg: Transgenic; \\ ELISA: Enzyme-linked immunosorbent assay; MWM: Morris water maze.}

\section{Competing interests}

The authors have no competing interests to declare.

\section{Authors' contributions}

$\mathrm{XL}$ acquired the data, analyzed and interpreted the data and drafted the manuscript. FG drafted the manuscript. QZ carried out the Morris water maze test. TH participated in the immunofluorescence assays. LL participated in drafting the manuscript. HW carried out the western blot test. QW handled funding and supervision, analyzed and interpreted the data, performed statistical analysis and drafted the manuscript. LX conceived and designed the research, handled funding and supervision and made critical revision of the manuscript for important intellectual content. All authors read and approved the final manuscript.

\section{Acknowledgments \\ This work was supported by the National Natural Science Foundation of China (Grant 81072888, 81035375, 81071052), the Overseas, Hong Kong \& Macao Scholars Collaborated Researching Fund (Grant 81228022), and the National Key Technology Research and Development Program of the Ministry of Science and Technology of China (2012BAI20B02).}

\section{Author details}

${ }^{1}$ Department of Anesthesiology, Xijing Hospital, The Fourth Military Medical University, Xi'an 710032, Shaanxi Province, China. ${ }^{2}$ Department of Anesthesiology, Stony Brook University School of Medicine, HSC L4 060, Stony Brook, NY 11794, USA.
Received: 18 May 2013 Accepted: 10 January 2014

Published: 22 January 2014

\section{References}

1. Reitz C, Brayne C, Mayeux R: Epidemiology of Alzheimer disease. Nat Rev Neurol 2011, 7:137-152.

2. Hampel H, Prvulovic D, Teipel S, Jessen F, Luckhaus C, Frölich L, Riepe MW, Dodel R, Leyhe T, Bertram L, Hoffmann W, Faltraco F, German Task Force on Alzheimer's Disease (GTF-AD): The future of Alzheimer's disease: the next 10 years. Prog Neurobiol 2011, 95:718-728.

3. Selkoe DJ: Images in neuroscience. Alzheimer's disease: from genes to pathogenesis. Am J Psychiatry 1997, 154:1198.

4. Chuang TT: Neurogenesis in mouse models of Alzheimer's disease. Biochim Biophys Acta 1802, 2010:872-880.

5. Henry RA, Hughes SM, Connor B: AAV-mediated delivery of BDNF augments neurogenesis in the normal and quinolinic acid-lesioned adult rat brain. Eur J Neurosci 2007, 25:3513-3525.

6. Larsen MH, Rosenbrock H, Sams-Dodd F, Mikkelsen JD: Expression of brain derived neurotrophic factor, activity-regulated cytoskeleton protein mRNA, and enhancement of adult hippocampal neurogenesis in rats after sub-chronic and chronic treatment with the triple monoamine re-uptake inhibitor tesofensine. Eur J Pharmacol 2007, 555:115-121.

7. Connor B, Young D, Yan Q, Faull RL, Synek B, Dragunow M: Brain-derived neurotrophic factor is reduced in Alzheimer's disease. Brain Res Mol Brain Res 1997, 49:71-81.

8. Siegel GJ, Chauhan NB: Neurotrophic factors in Alzheimer's and Parkinson's disease brain. Brain Res Brain Res Rev 2000, 33:199-227.

9. Feng $S$, Wang $Q$, Wang $H$, Peng $Y$, Wang L, Lu Y, Shi T, Xiong L: Electroacupuncture pretreatment ameliorates hypergravity-induced impairment of learning and memory and apoptosis of hippocampal neurons in rats. Neurosci Lett 2010, 478:150-155.

10. Wang Q, Peng Y, Chen S, Gou X, Hu B, Du J, Lu Y, Xiong L: Pretreatment with electroacupuncture induces rapid tolerance to focal cerebral ischemia through regulation of endocannabinoid system. Stroke 2009, 40:2157-2164.

11. Wang Q, Li X, Chen Y, Wang F, Yang Q, Chen S, Min Y, Li X, Xiong L: Activation of epsilon protein kinase $C$-mediated anti-apoptosis is involved in rapid tolerance induced by electroacupuncture pretreatment through cannabinoid receptor type 1. Stroke 2011, 42:389-396.

12. Tao J, Xue XH, Chen LD, Yang SL, Jiang M, Gao YL, Wang XB: Electroacupuncture improves neurological deficits and enhances proliferation and differentiation of endogenous nerve stem cells in rats with focal cerebral ischemia. Neurol Res 2010, 32:198-204.

13. Hwang IK, Chung JY, Yoo DY, Yi SS, Youn HY, Seong JK, Yoon YS: Effects of electroacupuncture at Zusanli and Baihui on brain-derived neurotrophic factor and cyclic AMP response element-binding protein in the hippocampal dentate gyrus. J Vet Med Sci 2010, 72:1431-1436.

14. Lu Z, Xiong L, Zhu Z, Wang Q, Chen M: Acupoint specificity of electroacupuncture preconditioning effect on cerebral ischemia injury in rats. Chin Acupunct Moxib 2002, 22:671-673.

15. Wimo A, Jönsson L, Gustavsson A, McDaid D, Ersek K, Georges J, Gulácsi L, Karpati K, Kenigsberg P, Valtonen $\mathrm{H}$ : The economic impact of dementia in Europe in 2008-cost estimates from the Eurocode project. Int J Geriatr Psychiatry 2011, 26:825-832.

16. McGowan E, Eriksen J, Hutton M: A decade of modeling Alzheimer's disease in transgenic mice. Trends Genet 2006, 22:281-289.

17. Masliah E, Sisk A, Mallory M, Mucke L, Schenk D, Games D: Comparison of neurodegenerative pathology in transgenic mice overexpressing V717F beta-amyloid precursor protein and Alzheimer's disease. J Neurosci 1996, 16:5795-5811.

18. Shi J, Perry G, Aliev G, Smith MA, Ashe KH, Friedland RP: Serum amyloid P is not present in amyloid beta deposits of a transgenic animal model. Neuroreport 1999, 10:3229-3232.

19. Aucoin JS, Jiang P, Aznavour N, Tong XK, Buttini M, Descarries L, Hamel E: Selective cholinergic denervation, independent from oxidative stress, in a mouse model of Alzheimer's disease. Neuroscience 2005, 132:73-86.

20. Bronfman FC, Moechars D, Van Leuven F: Acetylcholinesterase-positive fiber deafferentation and cell shrinkage in the septohippocampal pathway of aged amyloid precursor protein london mutant transgenic mice. Neurobiol Dis 2000, 7:152-168. 
21. Jing $X H$, Chen $S L$, Shi H, Cai H, Jin ZG: Electroacupuncture restores learning and memory impairment induced by both diabetes mellitus and cerebral ischemia in rats. Neurosci Lett 2008, 443:193-198.

22. Goate A, Chartier-Harlin MC, Mullan M, Brown J, Crawford F, Fidani L, Giuffra $L$, Haynes A, Irving N, James L: Segregation of a missense mutation in the amyloid precursor protein gene with familial Alzheimer's disease. Nature 1991, 349:704-706.

23. Cai XD, Golde TE, Younkin SG: Release of excess amyloid beta protein from a mutant amyloid beta protein precursor. Science 1993, 259:514-516.

24. Lopera F, Ardilla A, Martínez A, Madrigal L, Arango-Viana JC, Lemere CA, Arango-Lasprilla JC, Hincapí L, Arcos-Burgos M, Ossa JE, Behrens IM, Norton J, Lendon C, Goate AM, Ruiz-Linares A, Rosselli M, Kosik KS: Clinical features of early-onset Alzheimer disease in a large kindred with an E280A presenilin-1 mutation. JAMA 1997, 277:793-799.

25. Di Carlo M, Giacomazza D, San Biaqio PL: Alzheimer's disease: biological aspects, therapeutic perspectives and diagnostic tools. J Phys Condens Matter 2012, 24:244102.

26. Donovan MH, Yazdani U, Norris RD, Games D, German DC, Eisch AJ: Decreased adult hippocampal neurogenesis in the PDAPP mouse model of Alzheimer's disease. J Comp Neurol 2006, 495:70-83.

27. Gan L, Qiao S, Lan X, Chi L, Luo C, Lien L, Yan Liu Q, Liu R: Neurogenic responses to amyloid-beta plaques in the brain of Alzheimer's disease-like transgenic (pPDGF-APPSw, Ind) mice. Neurobiol Dis 2008, 29:71-80.

28. Choi SH, Veeraraghavalu K, Lazarov O, Marler S, Ransohoff RM, Ramirez JM, Sisodia SS: Non-cell-autonomous effects of presenilin 1 variants on enrichment-mediated hippocampal progenitor cell proliferation and differentiation. Neuron 2008, 59:568-580.

29. $\mathrm{Ma}$ QH, Futagawa $T$, Yang $W L$, Jiang XD, Zeng L, Takeda Y, Xu RX, Bagnard D, Schachner M, Furley AJ, Karagogeos D, Watanabe K, Dawe GS, Xiao ZC: A TAG1-APP signalling pathway through Fe65 negatively modulates neurogenesis. Nat Cell Biol 2008, 10:283-294.

30. Yu H, Saura CA, Choi SY, Sun LD, Yang X, Handler M, Kawarabayashi T, Younkin L, Fedeles B, Wilson MA, Younkin S, Kandel ER, Kirkwood A, Shen J: APP processing and synaptic plasticity in presenilin-1 conditional knockout mice. Neuron 2001, 31:713-726.

31. Thoenen H: Neurotrophins and neuronal plasticity. Science 1995, 270:593-598.

32. Durany N, Michel T, Kurt J, Cruz-Sánchez FF, Cervós-Navarro J, Riederer P: Brain-derived neurotrophic factor and neurotrophin-3 levels in Alzheimer's disease brains. Int J Dev Neurosci 2000, 18:807-813.

33. Holsinger RM, Schnarr J, Henry P, Castelo VT, Fahnestock M: Quantitation of BDNF mRNA in human parietal cortex by competitive reverse transcription-polymerase chain reaction: decreased levels in Alzheimer's disease. Brain Res Mol Brain Res 2000, 76:347-354.

34. Arancibia S, Silhol M, Moulière F, Meffre J, Höllinger I, Maurice T, TapiaArancibia L: Protective effect of BDNF against beta-amyloid induced neurotoxicity in vitro and in vivo in rats. Neurobiol Dis 2008, 31:316-326.

35. Tong L, Balazs R, Thornton PL, Cotman CW: Beta-amyloid peptide at sublethal concentrations downregulates brain-derived neurotrophic factor functions in cultured cortical neurons. J Neurosci 2004, 24:6799-6809.

36. Vitolo OV, Sant'Angelo A, Costanzo V, Battaglia F, Arancio O, Shelanski M: Amyloid beta -peptide inhibition of the PKA/CREB pathway and longterm potentiation: reversibility by drugs that enhance CAMP signaling. Proc Natl Acad Sci U S A 2002, 99:13217-13221.

37. Sairanen M, Lucas G, Ernfors P, Castren M, Castren E: Brain-derived neurotrophic factor and antidepressant drugs have different but coordinated effects on neuronal turnover, proliferation, and survival in the adult dentate gyrus. J Neurosci 2005, 25:1089-1094.

38. Wang $\mathrm{Q}$, Xiong L, Chen S, Liu Y, Zhu X: Rapid tolerance to focal cerebral ischemia in rats is induced by preconditioning with electroacupuncture: window of protection and the role of adenosine. Neurosci Lett 2005, 381:158-162.

doi:10.1186/1472-6882-14-37

Cite this article as: $\mathrm{Li}$ et al:: Electroacupuncture decreases cognitive impairment and promotes neurogenesis in the APP/PS1 transgenic mice. BMC Complementary and Alternative Medicine 2014 14:37.

\section{Submit your next manuscript to BioMed Central and take full advantage of:}

- Convenient online submission

- Thorough peer review

- No space constraints or color figure charges

- Immediate publication on acceptance

- Inclusion in PubMed, CAS, Scopus and Google Scholar

- Research which is freely available for redistribution

Submit your manuscript at www.biomedcentral.com/submit
Biomed Central 